\title{
A nonlinear finite element model of cartilage growth
}

\author{
Andrew Davol • Michael S. Bingham • Robert L. Sah • Stephen M. Klisch
}

\begin{abstract}
The long range objective of this work is to develop a cartilage growth finite element model (CGFEM), based on the theories of growing mixtures that has the capability to depict the evolution of the anisotropic and inhomogeneous mechanical properties, residual stresses, and nonhomogeneities that are attained by native adult cartilage. The CGFEM developed here simulates isotropic in vitro growth of cartilage with and without mechanical stimulation. To accomplish this analysis a commercial finite element code (ABAQUS) is combined with an external program (MATLAB) to solve an incremental equilibrium boundary value problem representing one increment of growth. This procedure is repeated for as many increments as needed to simulate the desired growth protocol. A case study is presented utilizing a growth law dependent on the magnitude of the diffusive fluid velocity to simulate an in vitro dynamic confined compression loading protocol run for 2 weeks. The results include changes in tissue size and shape, nonhomogeneities that develop in the tissue, as well as the variation that occurs in the tissue constitutive behavior from growth.
\end{abstract}

\section{Introduction}

Articular cartilage (AC) is a thin layer of connective tissue located within joints and on the ends of the long bones (Buckwalter and Mankin 1997) functioning as a low friction, wear-resistant,load-bearing material that facilitatesjoint motion (Maroudas and Venn 1977; Mow and Ratcliffe 1997). Adult cartilage is composed of a relatively small fraction of cells, called chondrocytes, within an extracellular matrix. The extracellular matrix is composed of water (60-90\%) and a solid matrix consisting mostly of a crosslinked collagen (COL) network and proteoglycans (PGs). AC has a poor intrinsic healing capacity that is likely related to its relatively low cellularity, metabolic activity, and avascularity (Buckwalter and Mankin 1998). Current clinical repair strategies for treating AC defects include tissue grafting and tissue engineering (Hunziker 2001; Smith et al. 2005). The attainment of a number of specific design goals related to tissue composition, structure, and function are likely to be critical to the development of a consistently successful strategy for the repair of AC defects.

The ultimate goal of this work is to develop an analytical model for growth of native and tissue engineered constructs of cartilage, especially to facilitate cartilage repair and regeneration. Growth as referred to in this work encompasses the phenomena of both growth, defined as the accretion of material similar to that already present, and remodeling, defined as the alteration of the endogenous or the overall material, leading to a change in the mechanical properties of the tissue. Differential growth of several tissue constituents can thus lead to growth and remodeling of the tissue as the mechanical properties will evolve during growth. The growth of an individual constituent can also include remodeling. For example, the crosslink density of the COL network may change as this constituent grows leading to a change in the mechanical properties of the COL constituent.

It has been shown that cartilage growth is dependent on biomechanical factors that are directly affected by local conditions such as stress, strain, or fluid velocities. These conditions themselves are in turn affected by the material 
properties which are evolving with growth. Continuum mechanics growth models attempt to close this "feedback loop". Since a stimulus is filtered by various tissue properties before signaling the cell nucleus to initiate mRNA production, continuum growth models attempt to model the temporal evolution of tissue composition and, consequently, biomechanical properties. For many years, bone-remodeling theories have been used to describe how a scalar measure of bone density and, consequently, the tissue's mechanical properties change in response to mechanical stimuli (Cowin and Hegedus 1976; Carter and Wong 1988; Beaupre et al. 1990; Cowin 1993). More recent theories have instead represented volumetric growth by a tensor quantity (Skalak et al. 1996, 1997) and were first used to study the growth of vascular tissues (Rodriguez et al. 1994; Taber and Eggers 1996; Taber 1998). In addition to our work on the development of cartilage growth mixture models, in recent years there has been much interest in the development of continuum growth models for single constituents (Chen and Hoger 2000; Epstein and Maugin 2000; DiCarlo and Quiligotti 2002; Lubarda and Hoger 2002; Kuhl and Steinmann 2003; Huang 2004; Volokh 2004; Lappa 2005; Menzel 2005), mixtures (Quiligotti 2002; Ganghoffer and Haussy 2005) and mixtures that employ a stress balance hypothesis (Humphrey and Rajagopal 2002; Preziosi and Farina 2002; Breward et al. 2003; Garikipati et al. 2004).

Various theories for the volumetric growth of elastic materials, as well as a cartilage growth mixture model (CGMM), have been proposed by Klisch and colleagues (Klisch et al. 2000, 2001, 2003, 2005b; Klisch and Hoger 2003). These theories allow for an elastic material to be composed of an arbitrary number of constituents that may grow and remodel independently. Growth for each constituent is described in terms of deformations that can be decomposed into growth deformations that model mass deposition and mechanical (elastic) deformations that ensure compatibility in the case of differential growth among the constituents. The notion of locally differential growth giving rise to residual stresses was suggested early by Skalak (1981), and then later by Chuong and Fung (1986), as an important feature of soft tissue growth.

Since the time scale for in vitro growth is several orders of magnitude greater than the time scale for applied mechanical loading, numerical approaches may be based on the solution to an incremental equilibrium growth boundary-value problem. This assumption is supported by our solution to the dynamic growth problem presented in Klisch et al. (2005b). We first proposed the incremental growth boundary-value problem in Klisch et al. (2001) and later modified it in Klisch (2006b) to be compatible with the finite element method.

Experiments with cartilage explants have quantified the metabolic response to mechanical stimuli such as hydrostatic pressure, fluid shear, and dynamic compression
(Hall et al. 1988; Sah et al. 1989; Guilak et al. 1997). A common testing protocol includes cyclic compression due to its effect of stimulating metabolism; in particular, cyclic compression of cartilage explants results in a frequencydependent stimulation of PG synthesis (Sah et al. 1989). Theoretical finite element model results suggest that PG synthesis in dynamic compression is best correlated with fluid diffusion velocity (fluid velocity relative to the solid matrix) (Kim et al. 1995; Buschmann et al. 1999). Due to its proven ability to stimulate synthesis in explants, dynamic compression has been a common in vitro protocol for mechanical stimulation of tissue-engineered constructs. Furthermore, PG and COL synthesis and accumulation have been positively correlated with fluid diffusion velocity in perfusion experiments with tissue engineered constructs grown in bioreactors in vitro (Pazzano et al. 2000; Davisson et al. 2002).

Biphasic models (Mow et al. 1980) have been derived from mixture theories (Atkin and Craine 1976), and represent the tissue as a permeable solid matrix (SM) and an inviscid fluid phase. For both phases, intrinsic incompressibility is assumed. Nonlinear biphasic models allow the permeability to depend on the strain of the tissue (Lai et al. 1981). Due to the nonhomogeneous structure of the tissue, relatively small physiological deformations of the type experienced in vivo may produce local large deformations within the tissue. Therefore, various finite deformation biphasic models have been proposed to account for the high levels of strain that may develop within the tissue. These models utilize a finite deformation constitutive law for the SM and apply them to the nonlinear biphasic model (Ateshian et al. 1997; Klisch et al. 2000). Poroelastic formulations are commonly included as a standard feature of commercial finite element analysis packages and have been successfully used for modeling soft tissues (Simon 1992). It has been shown that poroelastic models and biphasic models are equivalent if the fluid phase is considered inviscid (Simon 1992). Evaluations of the commercial finite element analysis package ABAQUS have shown that biphasic cartilage models can be successfully implemented using the soils consolidation feature of the software (Simon 1992; Wu et al. 1998).

The specific goal of the current research is to develop a CGFEM that has the capability to model the evolution of biomechanical properties during growth. Our current hypothesis is that the CGFEM is able to predict parameters that change during growth that are difficult to measure such as depthdependent biomechanical properties, which may then be used to identify specific parameters that should be measured experimentally, leading to the design of experiments that quantify tissue growth. The specific aims at this stage of the research were: (1) to solve the growth boundary value problem for an initially unstressed, homogeneous, isotropic specimen in confined compression for a 2 week period of growth, and (2) to analyze the model's ability to predict development of 
nonhomogeneity throughout the specimen, including constituent volume fractions for COL and PG, residual stresses and deformations, and the changing constitutive behavior as the tissue composition evolves.

\section{Methods}

The CGFEM is made up of two computational tools: an elemental growth routine (EGR) and a total specimen finite element model. These two tools interactively solve the equilibrium growth boundary value problem (BVP) as proposed in (Klisch et al. 2001; Klisch 2006b), subject to the growth theories presented in the CGMM (Klisch et al. 2000, 2003; Klisch and Hoger 2003). Since the time scale for each increment of in vitro growth is assumed to be 2-3 orders of magnitude greater than the time scale for in vitro loading, the dynamic effects of growth can be neglected (Klisch et al. 2005b), Thus, the dynamic BVP that characterizes the mechanical response to loading and the growth equilibrium BVP that characterizes the growth of individual elements are solved independently.

Some of the simplifying assumptions made in the CGMM are presented below, as they are also used in the CGFEM. Recall that the cartilage is modeled as a porous elastic SM that is composed of multiple constituents. The SM is saturated in an inviscid fluid that may flow through the SM. Based on this model of cartilage, the following assumptions are made regarding the growth of the material:

(i) The CGMM allows for an arbitrary number of SM constituents that are allowed to grow and remodel; however, the current work is limited to two growing constituents, PG and COL, and a third non-growing constituent referred to as "other" that describes the non-collagenous proteins.

(ii) All constituents are bound to the SM, and therefore the constituent deformation gradient tensors are equal to the SM deformation gradient tensor [this constraint can be relaxed to allow for mobile constituents (Klisch et al. 2003)].

(iii) The SM stress is equal to the sum of the PG and COL stresses.

(iv) Constituent stress constitutive equations are defined relative to reference configurations and only depend on mechanical (elastic) deformations.

(v) All growth is defined relative to a SM stress free configuration and can be decomposed into mechanical (elastic) and non-mechanical (growth) parts.

(vi) Growth occurs at constant stress and density for each constituent.

Several of the CGFEM's key capabilities are that it can predict the mechanical response due to in vitro loading, prescribe load-driven (stimulated) as well as non load-driven (unstimulated) growth, redefine the cartilage stress equation relative to any intermediate material configuration, and simulate in vitro growth for an arbitrary number of time increments (e.g., hours, days, weeks).

A diagrammatic representation of the equilibrium growth BVP over a specified time increment, $n$, is shown in Fig. 1. See Appendix A for details on the incremental growth problem. The time scale for $n$ is arbitrary; however, it is typically defined to be several orders of magnitude greater than the time scale of the applied biomechanical factors. For example, if $n$ is set to 1 day, during which a cartilage specimen may be cyclically loaded for part of that increment at a rate of $1 \mathrm{~Hz}$, the dynamic effects of loading (e.g., diffusive fluid velocity)
Fig. 1 A diagrammatic representation of the equilibrium growth BVP corresponding to increment $n$. The growth deformation during increment $\mathrm{n}$ for the unstressed solid matrix is given by ${ }_{n} \delta \boldsymbol{F}$ and the deformation required to equilibrate the entire specimen is given by ${ }_{n} \mathbf{F}_{\mathbf{c}}$

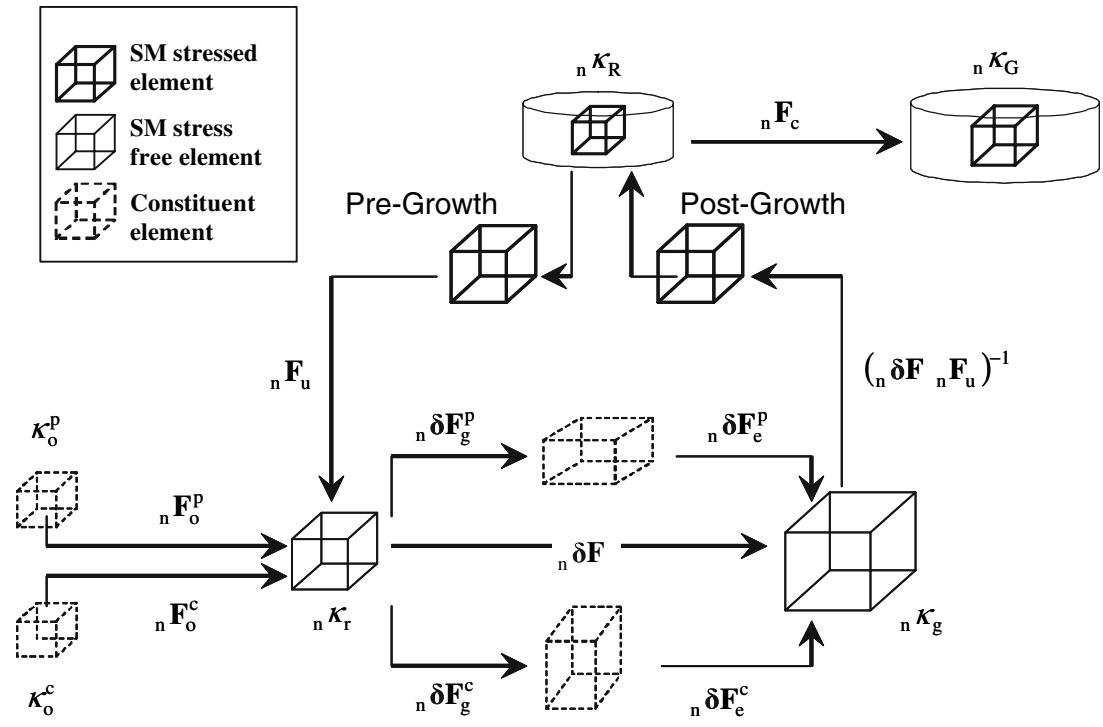


occur over a time period of $1 \mathrm{~s}$ as compared to the growth increment of 1 day. Also note that both stimulated and unstimulated growth are assumed to occur uniformly in an element with respect to time during $n$, and represent only a small change in the specimen's mass. Likewise, during growth, only small changes in the tissue's composition occur over a relatively large timescale, so it is reasonable to assume that dynamic effects like the strain rate of the growing tissue are also negligible (Klisch et al. 2005b). For these reasons, the growth BVP is treated as an equilibrium problem.

The overall growth BVP describes the tissue adaptation of the specimen from an initial configuration ${ }_{n} \kappa_{\mathrm{R}}$ to a grown configuration ${ }_{n} \kappa_{\mathrm{G}}$. The specimen is discretized into elements over which the mechanical properties are assumed to be homogeneous, although the specimen may be nonhomogenous. For the purposes of this description, assume that all biomechanical factors affecting growth for increment $n$ have already been determined.

The convention for specifying stress in a particular configuration, and stress functions relative to that configuration, are described with reference to Fig. 2. For example, the SM stress in configuration $\kappa$ is given as $\mathbf{T}_{\kappa}^{\mathbf{s}}$, and the SM stress function relative to the configuration $\kappa_{\mathrm{o}}$ is given as $\hat{\mathbf{T}}_{\kappa_{\mathbf{0}}}^{\mathbf{s}}$. The SM stress can be defined by the stress function through the elastic deformation $\mathbf{F}^{\mathbf{s}}$ as,

$\mathbf{T}_{\kappa}^{\mathbf{s}}=\hat{\mathbf{T}}_{\kappa_{\mathbf{0}}}^{\mathbf{s}}\left(\mathbf{F}^{\mathbf{s}}\right)$.

The material properties and the stress constitutive equations for the COL and PG are originally defined relative to their respective initial reference configurations $\left(\kappa_{\mathrm{o}}^{\mathrm{c}}, \kappa_{\mathrm{o}}^{\mathrm{p}}\right)$. For a constituent $\alpha$, the stress function relative to any configuration ${ }_{n} \kappa_{\beta}$, defined in the BVP, must be mapped to the constituent's initial reference configuration $\kappa_{\mathbf{o}}^{\alpha}$ where the material properties have been defined. For the BVP in Fig. 1, $\beta$ can represent $\mathrm{R}, \mathrm{r}, \mathrm{G}$, and $\mathrm{g}$, and $\alpha$ can represent $\mathrm{c}$ (COL) or $\mathrm{p}$ (PG). Since the stress constitutive equations only depend on elastic deformations, the stress function relative to ${ }_{n} \kappa_{\beta}$ will be a function of the residual elastic deformations and the elastic deformations that occur relative to ${ }_{n} \kappa_{\beta}$. Referring to Fig. 1 , the PG stress in configuration ${ }_{n} \kappa_{\mathrm{g}}$ can be described as

$\mathbf{T}_{n}^{\mathrm{p} \kappa_{\mathrm{g}}}=\hat{\mathbf{T}}_{n \kappa_{\mathrm{r}}}^{\mathrm{p}}\left({ }_{n} \mathbf{F}_{\mathrm{o}}^{\mathrm{p}},{ }_{n} \delta \boldsymbol{F}_{\mathrm{e}}^{\mathrm{p}}\right)$,

where ${ }_{n} \mathbf{F}_{\mathrm{O}}^{\mathrm{p}}$ is the residual elastic deformation that maps $\kappa_{\mathrm{o}}^{\mathrm{p}}$ to ${ }_{n} \kappa_{\mathrm{r}}$, and ${ }_{n} \delta \boldsymbol{F}_{\mathrm{e}}^{\mathrm{p}}$ is the incremental elastic deformation that

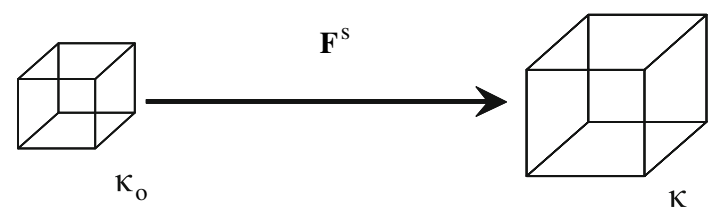

Fig. 2 Multiple configurations of a body described by the deformation $\mathrm{F}^{\mathrm{s}}$ maps ${ }_{n} \kappa_{\mathrm{r}}$ to ${ }_{n} \kappa_{\mathrm{g}}$. Appendix A describes this concept in more detail. Note that ${ }_{n} \delta \boldsymbol{F}_{\mathrm{g}}^{\mathrm{p}}$ is a non-elastic incremental growth tensor and does not directly affect the PG stress.

Using the assumptions listed above, the following paragraphs will describe the general procedure for solving the equilibrium growth BVP of Fig. 1 for an increment $n$. A generally stressed SM element in the pre-growth ${ }_{n} \kappa_{\mathrm{R}}$ configuration is unloaded to the element stress free configuration ${ }_{n} \kappa_{\mathrm{r}}$ through the deformation ${ }_{n} \mathbf{F}_{\mathrm{u}}$. For the initial increment, ${ }_{n} \mathbf{F}_{\mathrm{u}}=\mathbf{I}$, where $\mathbf{I}$ is the identity tensor, as the element is assumed to be stress free at the start of the simulation. For future increments, ${ }_{n} \mathbf{F}_{\mathrm{u}}$ is known from the history of the past increments.

Continuing with the description of the growth BVP solution from the SM stress free configuration ${ }_{n} \kappa_{\mathrm{r}}$, the element undergoes an incremental deformation ${ }_{n} \delta \boldsymbol{F}$ to the grown stress free configuration, ${ }_{n} \kappa_{\mathrm{g}}$. Both the COL and PG can experience unique incremental growth deformations $\left({ }_{n} \delta \boldsymbol{F}_{\mathrm{g}}^{\mathrm{c}},{ }_{n} \delta \boldsymbol{F}_{\mathrm{g}}^{\mathrm{p}}\right)$, also referred to as growth tensors. Therefore, in order to maintain the compatibility requirements imposed by assumption (ii), incremental constituent elastic deformations $\left({ }_{n} \delta \boldsymbol{F}_{\mathrm{e}}^{\mathrm{c}},{ }_{n} \delta \boldsymbol{F}_{\mathrm{e}}^{\mathrm{p}}\right)$ may develop, which are also referred to as compatibility tensors. Using assumptions (ii)-(vi), we can express the following relation,

${ }_{n} \delta F={ }_{n} \delta F_{\mathrm{e} n}^{\mathrm{c}} \delta F_{\mathrm{g}}^{\mathrm{c}}={ }_{n} \delta F_{\mathrm{e} n}^{\mathrm{p}} \delta F_{\mathrm{g}}^{\mathrm{p}}$,

where the total incremental growth deformation, ${ }_{n} \delta \boldsymbol{F}$, is unknown as are the constituent compatibility tensors. The constituent growth tensors are determined from the growth laws. The CGFEM solves for all of the unknowns in Eq. (3) by first solving for the compatibility tensors in terms of ${ }_{n} \delta \boldsymbol{F}$,

${ }_{n} \delta \boldsymbol{F}_{\mathrm{e}}^{\mathrm{c}}={ }_{n} \delta \boldsymbol{F}_{n} \delta \boldsymbol{F}_{g}^{\mathrm{c}^{-1}}$ and ${ }_{n} \delta \boldsymbol{F}_{\mathrm{e}}^{\mathrm{p}}={ }_{n} \delta \boldsymbol{F}_{n} \delta \boldsymbol{F}_{\mathrm{g}}^{\mathrm{p}^{-1}}$

The constituent stresses in configuration ${ }_{n} \kappa_{g}$ can then be determined from their stress functions relative to ${ }_{n} \kappa_{r}$ and are given as

$\mathbf{T}_{n \kappa_{\mathrm{g}}}^{\mathrm{c}}=\hat{\mathbf{T}}_{n \kappa_{\mathrm{r}}}^{\mathrm{c}}\left({ }_{n} \mathbf{F}_{\mathrm{o}}^{\mathrm{c}},{ }_{n} \delta \boldsymbol{F}_{n} \delta \boldsymbol{F}_{g}^{\mathrm{c}^{-1}}\right)$ and

$\mathbf{T}_{n}^{\mathrm{p} \kappa_{\mathrm{g}}}=\hat{\mathbf{T}}_{n}^{\mathrm{p} \kappa_{\mathrm{r}}}\left({ }_{n} \mathbf{F}_{\mathrm{o}}^{\mathrm{p}},{ }_{n} \delta \boldsymbol{F}_{n} \delta \boldsymbol{F}_{g}^{\mathrm{p}^{-1}}\right)$.

Recalling assumption (iii) and also noting that the SM stress, $\mathbf{T}_{n \kappa_{\mathrm{g}}}^{\mathrm{s}}$, is equal to zero, we obtain:

$\mathbf{T}_{n \kappa_{\mathrm{g}}}^{\mathrm{c}}+\mathbf{T}_{n \kappa_{\mathrm{g}}}^{\mathrm{p}}=\mathbf{0}$,

which can be solved for the incremental growth deformation ${ }_{n} \delta \boldsymbol{F}$. It can be seen from Fig. 1 that growth in the current model defines the evolution of the solid matrix stressfree configuration, as proposed in Rodriguez et al. (1994), which leads to experimental prescriptions for the elastic and growth tensors via destructive experiments that relieve residual stress, as discussed in Klisch et al. (Klisch et al. 2003, 2006). The solution to these equations is accomplished inside 
MATLAB. With this result and Eqs. (4), the constituent compatibility tensors are solved for.

The final stages of solving the growth BVP involve deforming the grown SM element in configuration ${ }_{n} \kappa_{\mathrm{g}}$ to the stressed post-growth configuration ${ }_{n} \kappa_{\mathrm{R}}$ through the deformation $\left({ }_{n} \delta \boldsymbol{F}_{n} \mathbf{F}_{\mathrm{u}}\right)^{-\mathbf{1}}$. Note that the pre-growth and post-growth ${ }_{n} \kappa_{\mathrm{R}}$ configurations are equivalent in the sense that every material point occupies the same location in each configuration. The difference between the configurations is the stress state of the element, and the SM stress constitutive equation for deformations out of this configuration.

This procedure is carried out for each element of the entire tissue specimen. Generally, the growth of each element will vary based on the growth laws and variations of the biomechanical factors throughout the entire specimen. Therefore, in general, the elements of the specimen will not be in equilibrium in the post-growth configuration ${ }_{n} \kappa_{\mathrm{R}}$. The final step in solving the growth BVP is to solve for the nonhomogeneous deformation ${ }_{n} \mathbf{F}_{\mathrm{c}}$, which ensures equilibrium throughout the specimen for the grown configuration ${ }_{n} \kappa_{\mathrm{G}}$. This solution is accomplished with the finite element model (ABAQUS).

Subsequent increments are handled the same as the first with the exception that the initial unloading deformation is no longer trivial. However, it can be seen from Fig. 1 that the deformation required to unload the element is known from the previous increments, ${ }_{n} \mathbf{F}_{\mathrm{u}}={ }_{\mathrm{n}-1} \delta \boldsymbol{F}_{\mathrm{n}-1} \mathbf{F}_{\mathrm{u} \mathrm{n}-1} \mathbf{F}_{\mathrm{c}}^{-1}$.

\section{Analysis}

This section presents a growth simulation case study using the CGFEM. One of the long-term goals of this work includes determining the constituent growth laws and other material and growth parameters for cartilage. Since these parameters are currently unknown, they are assumed for the analyses presented below. This case study will consider small and equal amounts of unstimulated COL and PG growth, with increased amounts of stimulated PG growth. All assumed parameters are based on physically relevant values and/or observations, but their absolute validity is unknown. Therefore, the results from these analyses should be physically relevant; however, the accuracy of the results will have no basis for comparison, and will not be investigated quantitatively.

\subsection{Modeling parameters}

The simulation modeled stimulated and unstimulated in vitro growth of a cartilage disk specimen subjected to a confined compression loading protocol as shown in Fig. 3. The specimen was modeled as an initially stress-free homogenous bovine cartilage explant $1 \mathrm{~mm}$ thick and $3 \mathrm{~mm}$ in diameter. The specimen is modeled as bovine due to the availability of bovine material properties in the literature, and because future growth experiments are planned using bovine specimens. Only one quarter of the top half of the specimen was modeled in the simulation taking advantage of the symmetry of the problem.

Growth and loading was simulated for a 2-week period, divided into fourteen 1-day increments. Therefore, the equilibrium growth BVP was solved 14 times for each element (the model presented here utilized 3,000 elements), once for each daily growth increment. At the end of the 2-week growth period the specimen was released from the confining chamber to determine the new configuration of the grown specimen without the presence of the radial confinement.

\subsection{Material characterization and parameters}

An isotropic nonlinear bimodular constitutive equation with five material constants was used for the SM in this analysis. Several different models of this type have been investigated and found to provide reasonably accurate predictions of cartilage in confined compression, unconfined compression, and tension (Klisch 2006a). The current model is a simplified version of those studied previously with the collagen modeled as a compressible Neo-Hookean material (Schroder and Neff 2003) (see Appendix B for more details on bimodular materials). For example, the SM stress equation that describes the constitutive behavior of the specimen as growth
Fig. 3 Schematic of confined compression apparatus for tissue growth and loading experiments

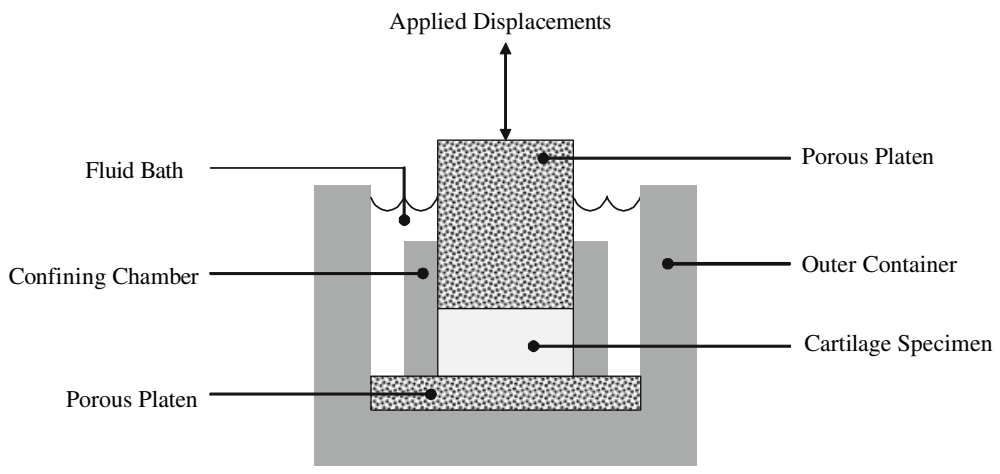


and remodeling occur during the first day of growth is

$$
\begin{aligned}
\hat{\mathbf{T}}_{1 \kappa_{\mathrm{r}}}^{\mathbf{s}}= & \frac{1}{J^{c}}\left[\mu\left(\mathbf{B}^{\mathrm{c}}-\mathbf{I}\right)+\frac{1}{2} \lambda\left[J^{c}\right]\left(\left(J^{c}\right)^{2}-1\right) \mathbf{I}\right] \\
& -\left[\alpha_{1}\left(\operatorname{det} \mathbf{F}_{\mathrm{e}}^{\mathrm{p}}\right)^{-1}+\alpha_{2}\left(\operatorname{det} \mathbf{F}_{\mathrm{e}}^{\mathrm{p}}\right)^{-2}\right] \mathbf{I} .
\end{aligned}
$$

In this relation, $\mathbf{B}^{\mathrm{c}}$ is the left Cauchy-Green deformation tensor for the collagen $\left(\mathbf{B}^{\mathrm{c}}=\mathbf{F}_{\mathrm{e}}^{\mathrm{c}} \mathbf{F}_{e}^{\mathrm{c}^{\mathrm{T}}}\right), J^{\mathrm{c}}$ is the determinant of the collagen deformation gradient $\left(J^{\mathrm{c}}=\operatorname{det}\left(\mathbf{F}_{\mathrm{e}}^{\mathrm{c}}\right)\right)$, and $\mu, \lambda, \alpha_{1}$, and $\alpha_{2}$, are material constants. Note that for this first day ${ }_{1} \kappa_{\mathrm{r}}={ }_{1} \kappa_{\mathbf{R}}$ since the specimen was initially stress free. The values for the material constants are given in Table 1. These values are based on bovine data found in the literature (Klisch et al. 2005a,b).

The compatibility tensors $\mathbf{F}_{\mathrm{e}}^{\mathrm{c}}$ and $\mathbf{F}_{\mathrm{e}}^{\mathrm{p}}$ are defined relative to the stress free pre-growth reference configuration ${ }_{n} \kappa_{\mathrm{r}}$, but recall that the total stress also requires the history of the deformations relative to the reference configurations $\kappa_{\mathrm{o}}^{\mathrm{c}}$ and $\kappa_{\mathrm{O}}^{\mathrm{p}}$ (see Appendix A).

The initial constituent residual elastic deformation tensors required to put the solid matrix in equilibrium $\left(\mathbf{T}_{1 \kappa_{\mathrm{r}}}^{\mathrm{s}}=\mathbf{0}\right)$ are given as:

${ }_{1} \mathbf{F}_{\mathrm{o}}^{\mathrm{c}}=(1.05) \mathbf{I}$,

${ }_{1} \mathbf{F}_{\mathrm{o}}^{\mathrm{p}}=\mathbf{I}$.

Recall that these residual elastic deformations are combined with the SM elastic deformations that develop as growth and loading occur to calculate the total COL and PG deformation gradients, $\mathbf{F}_{\mathrm{e}}^{\mathrm{c}}$ and $\mathbf{F}_{\mathrm{e}}^{\mathrm{p}}$, used in Eq. (7).

The model is run in ABAQUS using the poroelastic formulation which allows for variable permeability throughout the loading. The material permeability function is given as (Argoubi and Shirazi-Adl 1996; Ateshian et al. 1997)

$k=k_{\mathrm{o}}\left[\frac{e}{e_{\mathrm{o}}}\right]^{2} \exp \left[\frac{M}{2}\left(\left\{\frac{1+e}{1+e_{\mathrm{o}}}\right\}^{2}-1\right)\right]$,

with $k_{\mathrm{o}}$ the permeability in the absence of strain, $e$ the void ratio, $e_{\mathrm{o}}$ the initial void ratio, and $M$ the non-dimensional permeability coefficient. Listed in Table 2 are the constant value parameters found in Eq. (9). These values were also determined or compiled from bovine data in the literature. Note that the permeability is listed in the form required by ABAQUS which is derived from

$k_{\mathrm{o}}=\bar{k}_{\mathrm{o}} \rho_{\mathrm{w}} g$,

Table 1 Material properties for five parameter material model

\begin{tabular}{lllll}
\hline$\lambda_{+}(\mathrm{MPa})$ & $\lambda_{-}(\mathrm{MPa})$ & $\mu(\mathrm{MPa})$ & $\alpha_{1}(\mathrm{MPa})$ & $\alpha_{2}(\mathrm{MPa})$ \\
\hline 0.35 & 0.02 & 0.04 & 0.01 & 0.045 \\
\hline
\end{tabular}

Note: $\lambda\left[J^{\mathfrak{c}}\right]=\lambda_{+}$if $J^{\mathfrak{c}} \geq 1, \lambda\left[J^{\mathfrak{c}}\right]=\lambda_{-}$if $J^{\mathfrak{c}}<1$
Table 2 Permeability parameters

\begin{tabular}{llll}
\hline$M$ & $\bar{k}_{\mathrm{o}} \mathrm{m}^{4} / \mathrm{Ns}$ & $k_{\mathrm{o}} \mathrm{mm} / \mathrm{s}$ & $e_{\mathrm{o}}$ \\
\hline 2.2 & $2.7 \times 10^{-15}$ & $2.64 \times 10^{-8}$ & 7.13 \\
\hline
\end{tabular}

Table 3 Initial volume fractions

\begin{tabular}{lllll}
\hline$\varphi_{\mathrm{o}}^{\mathrm{c}}$ & $\varphi_{\mathrm{o}}^{\mathrm{p}}$ & $\varphi_{\mathrm{o}}^{\mathrm{o}}$ & $\varphi_{\mathrm{o}}^{\mathrm{s}}$ & $\varphi_{\mathrm{o}}^{\mathrm{f}}$ \\
\hline 0.072 & 0.013 & 0.038 & 0.123 & 0.877 \\
\hline
\end{tabular}

where $\bar{k}_{\mathrm{o}}$ is the permeability in $\frac{\mathrm{m}^{4}}{\mathrm{Ns}}, \rho_{\mathrm{w}}$, is the density of the wetting liquid, and $g$ is the acceleration of gravity.

The initial void ratio, $e_{\mathrm{o}}$, is given as

$e_{\mathrm{o}}=\frac{\phi_{\mathrm{o}}^{\mathrm{f}}}{\phi_{\mathrm{o}}^{\mathrm{s}}}$

Note that $\phi_{\mathrm{o}}^{\mathrm{s}}$ is the sum of initial SM constituent volume fractions and $\phi_{\mathrm{o}}^{\mathrm{f}}$ is the fluid volume fraction. The initial fluid volume fraction is derived from the incompressibility constraint

$\phi_{\mathrm{o}}^{\mathrm{s}}+\phi_{\mathrm{o}}^{\mathrm{f}}=1$.

The assumed initial volume fractions including PG, COL, and other (o) are listed in Table 3.

\subsection{FE loading and stimulated growth trigger}

For the considered growth case, stimulated growth for each constituent was assumed to depend on the magnitude of the diffusive fluid velocity. Based on work by Buschmann et al. (1999), growth appears to be stimulated in regions of the specimen where the magnitude of the diffusive fluid velocity exceeds the value $2.5 \times 10^{-4} \mathrm{~mm} / \mathrm{s}$. Therefore, this value was defined in the CGFEM such that if, during loading, any element in the specimen experienced a diffusive fluid velocity above this value, that element was subject to stimulated growth as defined by the growth laws.

Obviously the loading and boundary conditions, coupled with the permeability, regionally affects the magnitude of the diffusive fluid velocity throughout the specimen. Since only confined compression loading was considered for this case study, variations in this parameter only occurred axially, with zero velocity at the mid-plane, and maximum velocity occurring symmetrically at the top and bottom surfaces.

To achieve a diffusive velocity distribution that stimulated nonhomogeneous axial growth, the specimen was loaded in confined compression by applying a $0.1 \mathrm{~mm}$ displacement to the top surface at the rate of $2.5 \times 10^{-3} \mathrm{~mm} / \mathrm{s}$. Note that relative to the specimen mid-plane, where the diffusive fluid velocity is zero, the top and bottom surfaces were displaced at the rate $1.25 \times 10^{-3} \mathrm{~mm} / \mathrm{s}$, which equals the magnitude of 
Table 4 Growth tensors for case study

\begin{tabular}{llll}
\hline $\begin{array}{l}\text { Collagen } \\
\text { unstimulated }\end{array}$ & $\begin{array}{l}\text { Collagen } \\
\text { stimulated }\end{array}$ & $\begin{array}{l}\text { Proteoglycan } \\
\text { unstimulated }\end{array}$ & $\begin{array}{l}\text { Proteoglycan } \\
\text { stimulated }\end{array}$ \\
\hline${ }_{n} \delta \boldsymbol{F}_{\mathrm{g}}^{\mathrm{c}}=(1.01)^{1 / 3} \mathbf{I}$ & ${ }_{n} \delta \boldsymbol{F}_{\mathrm{g}}^{\mathrm{c}}=(1.01)^{1 / 3} \mathbf{I}$ & ${ }_{n} \delta \boldsymbol{F}_{\mathrm{g}}^{\mathrm{p}}=(1.01)^{1 / 3} \mathbf{I}$ & ${ }_{n} \delta \boldsymbol{F}_{\mathrm{g}}^{\mathrm{p}}=(1.03)^{1 / 3} \mathbf{I}$ \\
\hline
\end{tabular}

the diffusive fluid velocity at these surfaces. This loading rate ensured that the outer regions of the specimen experienced stimulated growth, since the diffusive fluid velocity in these regions always exceeded the minimum trigger value, and the middle of the specimen experienced no stimulated growth since the diffusive fluid velocity was zero in this region. This method was chosen to demonstrate the capabilities of the model, not to attempt to match experimental protocols.

Each element in the specimen experienced unstimulated COL and PG mass deposition (growth), at the rate of $1 \%$ per day. For elements where stimulated growth was triggered, an additional 2\% stimulated PG growth occurred. The growth tensors associated with this case study are shown in Table 4 .

The reader should note that the growth laws for stimulated growth do not account for the excess magnitude of the diffusive velocity above the trigger value, or the time duration of the trigger event. For example, if an element experienced a maximum diffusive fluid velocity value equal to the trigger value for just one instant in time, it was prescribed the same amount of stimulated growth as an element that experienced a maximum diffusive fluid velocity equal to five times the trigger value for the entire loading duration. This off-on nature of the growth law can be easily modified in this model as more data become available.

\section{Results}

This section describes the tissue configuration resulting from the simulation in several ways. The physical change in the dimensions of the tissue is tracked by monitoring the height of the specimen throughout the simulation and observing the final variation in the radius of the tissue after removal of the radial confinement at the end of the 14 days of growth. The composition of the tissue is expressed as the variation in the volume fractions of the PGs and COL through the height of the specimen. The stresses generated due to the differential growth of the constituents are also reported. Finally, the constitutive behavior of elements in the center and on the surface of the specimen is compared at the end of the simulation. All these characteristics are factors that may help to design future protocols for controling the growth of cartilage.

The daily change in height of the specimen inside the confining chamber can be seen in Fig. 4. The daily growth increases as we would expect since the growth law is based on a percentage increase of the existing mass. It can be seen

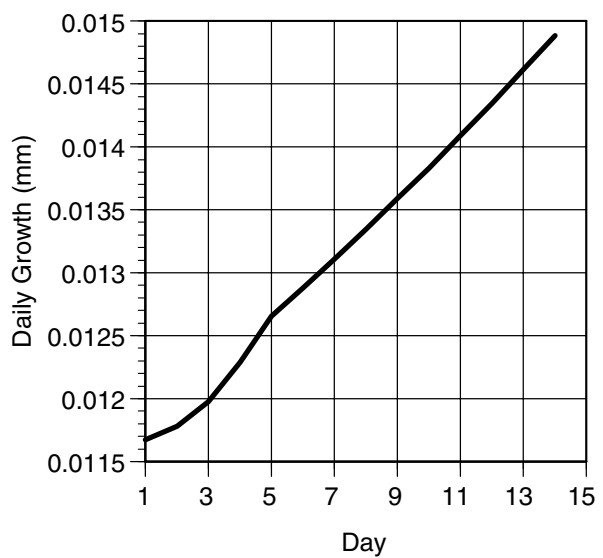

Fig. 4 Daily change in specimen height inside the confining chamber

from Fig. 4 that there is acceleration in the growth in the early stages of the simulation. This occurs due to the off-on nature of the stimulated growth law used for this study. Between days 2 and 5 enough growth has occurred so that an additional row of elements reaches the threshold fluid velocity and begins to see stimulated growth, therefore accelerating the daily growth rate. The shape of the released specimen can be used to help establish and verify appropriate growth laws by comparing to experimental results. The varying radius of the released specimen is shown in Fig. 5. The top surface where more PG deposition was occurring has an increased radius relative to the center of the specimen.

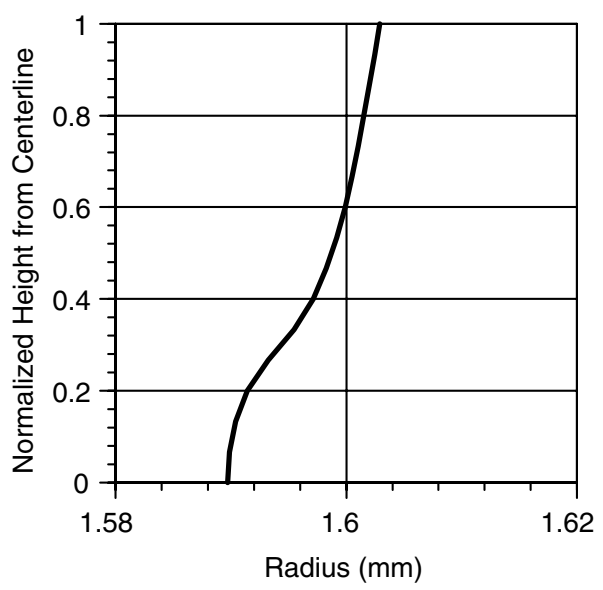

Fig. 5 Radius vs. height for released specimen after 14 days of growth with increased PG growth in the material away from the centerline (initial radius $1.5 \mathrm{~mm}$ ) 


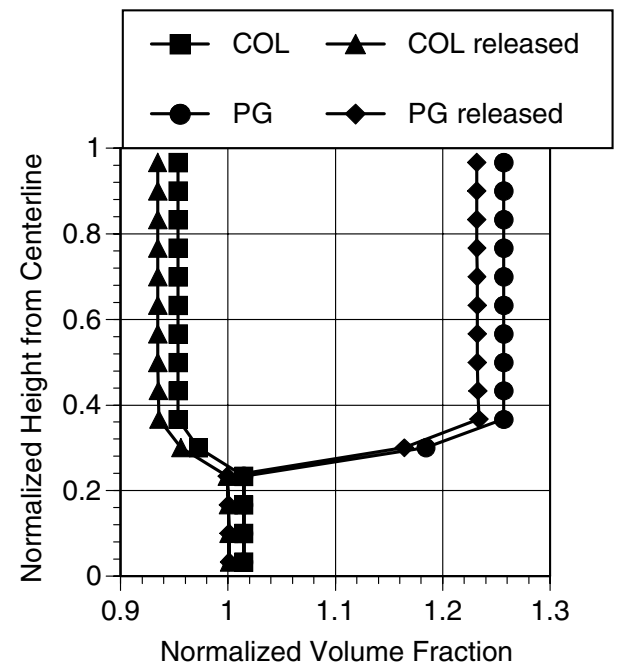

Fig. 6 Volume fractions of the PG and COL normalized to the day zero values, after 14 days of growth, before and after release from the confining chamber

As differential growth occurs between the constituents, non-homogeneity begins to be seen in the model. Fig. 6 shows the constituent volume fractions after 14-days of growth, before and after release from the confining chamber, normalized by their initial values. As would be expected, the regions seeing stimulated growth now have a higher volume fraction of PG.

Residual stresses can also be investigated in the confined and released configurations as shown in Fig. 7. In the confining chamber the areas of increased PG deposition near the surface show increased radial compressive stresses compared to the areas of the tissue near the centerline of the specimen that underwent less overall growth. When released the entire disk expands but the regions near the mid-plane, which did not see as much PG deposition, do not want to expand as much as the surface regions, leading to the residual stress state seen in Fig. 7. Notice that the material near the midplane is now in tension holding back the material near the surface. It can also be seen that the stresses are an order of magnitude lower after release from the confining chamber.

As the constituents grow differentially throughout the simulation the constitutive behavior of the tissue is changing.
The ability of the model to capture this behavior is evident in Fig. 8 where the stress strain behavior in the axial direction is compared for an element on the top surface of the specimen with an element on the specimen mid-plane. The area on the top surface with more PG growth throughout the simulation now exhibits stiffer behavior in compression. Also note the bimodular nature of the material is evident as the material softens when the collagen network goes into compression.

The computational resources for this simulation were fairly modest. The current model (Fig. 7) has $\sim 15,000$ degrees of freedom, which for the current material model takes approximately $25 \mathrm{~min}$ per growth increment with a single $1.8 \mathrm{GHz}$ processor and $1 \mathrm{~GB}$ of Ram.

Studies were done to validate the independence of the results presented to the choice of the growth increment. Simulations were repeated with a half day growth increment and the appropriately scaled growth tensors and no difference was seen in the final composition or stress state of the grown models.

\section{Discussion}

The case study presented above demonstrates the ability of the CGFEM to predict evolving nonhomogeneous composition of a cartilage explant during in vitro growth in a confining chamber subject to dynamic compression. This result is significant due to the fact that adult native cartilage displays just such a highly nonhomogenous structure that may be necessary to ensure a successful repair strategy using tissue engineered constructs. The volume fractions are one measure of the nonhomogenous nature of the grown specimen but the CGFEM also predicts the evolution of the stress strain response as the solid matrix grows and remodels. The material response curves depicted in Fig. 8 demonstrate how the material stiffens in compression as the PG volume fraction increases. This ability is essential to achieve the goal of simulating the evolution of cartilage constructs since the growth is believed to be dependent on the material's mechanical response to loading.

The confined compression protocol has several advantageous qualities that made it a good choice for the case study
Fig. 7 Distribution of the solid matrix Cauchy stress in the 1 direction after 14 days of growth before and after release from the confining chamber. The residual stresses and nonuniform radial expansion demonstrate the effect of differential growth
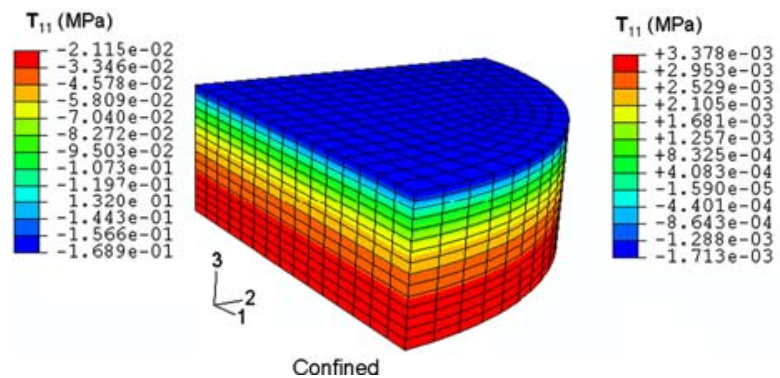

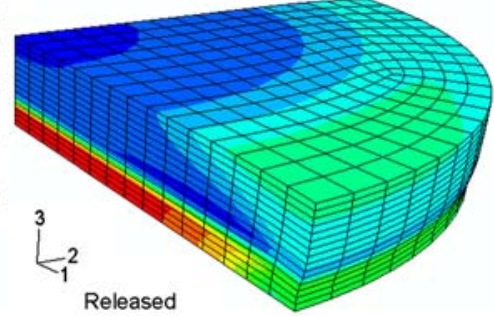




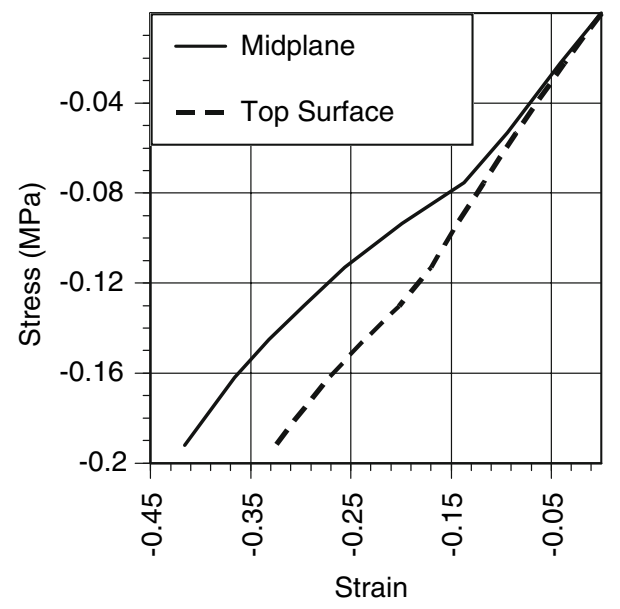

Fig. 8 Comparison of the SM unconfined compressive stiffness at the surface and mid-plane of the specimen on day-14 demonstrating the evolution of the material elastic behavior with growth

presented in this work. The fluid velocity profile for this protocol is quite simple in that the fluid velocity only has a component in the axial direction and the velocity is known to be zero at the mid-plane due to the symmetry of the problem. This ensures that if the rate of compression is chosen carefully the outer layers of tissue are guaranteed to see stimulated growth whereas the tissue on the mid-plane will not. Also the tissue deformations in this test protocol do not include any rotations until the tissue is released from the confining chamber. This allowed for several simple material models to be evaluated against closed form solutions before more complex models were implemented.

This initial work has demonstrated many of the capabilities of the CGFEM but the study was intentionally limited at this stage of the research. There are no material rotations until the specimen is released which simplifies the solution of the boundary value problem for the growth deformations. As deformations become more complex, solving for the growth deformation tensor may become more difficult. As can be seen by the selection of the material model, currently the material is assumed to be isotropic. This limitation does not reflect the true nature of native cartilage which has been found to display anisotropic behavior (Wang et al. 2003; Ficklin et al. 2006). Future work will extend the bimodular stressstrain equation for the collagen constituent to include anisotropic behavior (Klisch 2006a). Also, it has been suggested that to accurately model specific growth experiments a collagen remodeling parameter (related to collagen-specific crosslink or fibril binding molecules) should be included that changes the mechanical properties of the collagen constituent (Klisch et al. 2005a).

One area of great promise for the CGFEM is the ability of the model to simulate experimental protocols. Such simulations experiments can be specifically designed to give needed results with much less trial and error in the lab. It is emphasized that accurate growth laws are required for this model to mature into a truly useful tool for simulating experimental protocols for cartilage growth. Currently, experiments are being planned to accomplish this aim. Specifically steady-state perfusion experiments are being designed to help quantify the growth tensors.

Although no attempt is presently made to compare the predicted growth to experimental data, available data have been used wherever possible to calibrate the model. The fluid velocity used to trigger stimulated growth is based on experimental observations (Buschmann et al. 1999). The off-on nature of the stimulated growth trigger can be easily modified to account for dose dependence if future experiments display such behavior. Although fluid velocity was chosen as the trigger for the current case study, the model is general enough to allow for many different mechanical stimuli to be used. The material constants were all chosen based on available data (Klisch et al. 2003; Chahine et al. 2004).

The capabilities of the CGFEM evident in the results presented above illustrate that this model may be capable of predicting nonhomogeneous growth of cartilage constructs for use in tissue engineering applications. One advantage of the CGFEM is that the analytical modeling will be less timeconsuming, and consequently less expensive, than experimentation. Currently, these types of quantitative growth models are sparse in the field of cartilage tissue engineering. As a specific example of the clinical significance of this work, consider the difficulty in the engineering of target constructs that meet specific compositional, structural, and functional requirements with the aim of repairing cartilage defects. It is possible that, in future, a construct's requirements for a specific anatomic site may be identified by noninvasive methods such as MRI or ultrasound. Indeed, quantitative MRI parameters have been shown to explain up to $87 \%$ of the variation in specific mechanical properties (Nieminen et al. 2004) and the structural, compositional, and mechanical variations in degenerate and normal cartilage (Nissi et al. 2004). If the CGFEM can be calibrated for a tissue engineering protocol within a range of in vitro biochemical and biomechanical stimuli for a specific cell population, then it may be used to predict the in vitro protocol needed to produce a construct from a nearly homogenous state to one that more closely resembles the native tissue state with its depth-dependent nonhomogeneties.

Acknowledgments Funding was received from the National Science Foundation and the Office of Naval Research (Bingham, Klisch and Davol), the National Science Foundation and the National Institute of Health (Sah), and the Donald E. Bentley Center for Innovation (Klisch).

\section{Appendix A: Incremental growth analysis}

In this appendix, we discuss the derivation of an equation (see A4) that describes how the total and incremental 


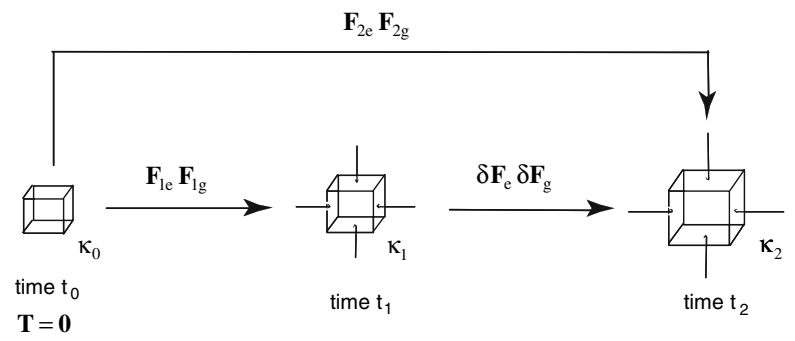

Fig. 9 Schematic of the incremental growth boundary-value-problem

elastic deformation gradient tensors are related for a growing elastic material which serves as the foundation for the computational solution. This result was first presented in Klisch (2006b). Consider a subset of the body that is unloaded and stress-free in a reference configuration $\kappa_{\mathrm{o}}(P)$ at time $t_{\mathrm{o}}$ (Fig. 9). The subset represents a finite element in the computational setting. Suppose that the deformation gradient tensor $\mathbf{F}_{1}=\mathbf{F}_{\mathbf{1 e}} \mathbf{F}_{1 \mathrm{~g}}$ maps $\kappa_{\mathrm{o}}(P)$ to the configuration $\kappa_{1}(P)$ and is known at time $t_{1}$. Also, let the incremental deformation gradient tensor $\delta \boldsymbol{F}$ that maps $\kappa_{1}(P)$ to $\kappa_{2}(P)$ at time $t_{2}$ obey the decomposition

$\delta \boldsymbol{F}=\delta \boldsymbol{F}_{\mathrm{e}} \delta \boldsymbol{F}_{\mathrm{g}}$,

where $\delta \boldsymbol{F}_{\mathrm{e}}$ and $\delta \boldsymbol{F}_{\mathrm{g}}$ are the incremental elastic and growth tensors. The tensor $\delta \boldsymbol{F}_{\mathrm{g}}$ quantifies the amount and orientation of mass deposition relative to $\kappa_{1}(P)$ and the tensor $\delta \boldsymbol{F}_{\mathrm{e}}$ ensures compatibility of the body. Finally, suppose that the deformation gradient tensor $\mathbf{F}_{2}=F_{2 \mathbf{e}} \mathbf{F}_{2 \mathrm{~g}}$ maps $\kappa_{\mathrm{0}}(P)$ to $\kappa_{2}(P)$ at time $t_{2}$. Using the chain rule, we obtain

$\mathbf{F}_{2}=\delta \boldsymbol{F} \boldsymbol{F}_{1} \Rightarrow \mathbf{F}_{2}=\delta \boldsymbol{F}_{\mathrm{e}} \delta \boldsymbol{F}_{\mathrm{g}} \mathbf{F}_{1 \mathrm{e}} F_{1 \mathrm{~g}}$.

The solution to the incremental growth problem involves determining $\boldsymbol{\delta} \boldsymbol{F}_{\mathrm{g}}$ and $\boldsymbol{\delta} \boldsymbol{F}_{\mathrm{e}}$.

The biological aspects of growth suggest that a growth law should be defined relative to $\kappa_{1}(P)$, which is considered as the reference configuration for the incremental growth problem. The growth law corresponds to the time rate of change of the incremental growth tensor $\delta \boldsymbol{F}_{\mathrm{g}}$. In Klisch (2006b), we derived an equation for determining $\delta \boldsymbol{F}_{\mathrm{g}}$ from the growth law using a first-order Taylor series expansion. Once $\delta \boldsymbol{F}_{\mathrm{g}}$ is known, the equilibrium equation $\operatorname{div}^{\mathbf{s}}=\mathbf{0}$ and the stress constitutive equation are used to determine $\delta \boldsymbol{F}_{\mathrm{e}}$ for $P$ while satisfying the boundary conditions on the body $B$. However, determining the manner in which the stress constitutive equation evolves is not trivial, since the stress is assumed to depend only on the elastic deformations. Suppose that the stress constitutive functions for deformations out of $\kappa_{0}(P)$ and $\kappa_{1}(P)$ are of the general forms $\hat{\mathbf{T}}_{\kappa_{0}}\left(\mathbf{F}_{2 \mathrm{e}}\right)$ and $\hat{\mathbf{T}}_{\kappa_{1}}\left(\boldsymbol{\delta} \boldsymbol{F}_{\mathrm{e}}\right)$, respectively. Usually, the function $\hat{\mathbf{T}}_{\kappa_{0}}\left(\mathbf{F}_{2 \mathrm{e}}\right)$ will be known; consequently, we need to determine $\hat{\mathbf{T}}_{\kappa_{1}}\left(\delta \boldsymbol{F}_{\mathrm{e}}\right)$. The physical idea that must be satisfied is that the stress in $\kappa_{2}(P), \mathbf{T}_{\kappa_{2}}$, must be the same when evaluated using either of these stress constitutive functions:

$\mathbf{T}_{\kappa_{2}}=\hat{\mathbf{T}}_{\kappa_{0}}\left(\mathbf{F}_{2 \mathrm{e}}\right)=\hat{\mathbf{T}}_{\kappa_{1}}\left(\boldsymbol{\delta} \boldsymbol{F}_{\mathrm{e}}\right)$.

For orthotropic, transversely isotropic, and isotropic materials, if $\mathbf{F}_{2 \mathbf{e}}$ and $\hat{\mathbf{T}}_{\kappa_{1}}\left(\boldsymbol{\delta} \boldsymbol{F}_{\mathrm{e}}\right)$ satisfy

$\mathbf{F}_{2 \mathrm{e}}=\delta \boldsymbol{F}_{\mathrm{e}} \mathbf{F}_{1 \mathrm{e}}, \hat{\mathbf{T}}_{\kappa_{1}}\left(\boldsymbol{\delta} \boldsymbol{F}_{\mathrm{e}}\right)=\hat{\mathbf{T}}_{\kappa_{0}}\left(\boldsymbol{\delta} \boldsymbol{F}_{\mathrm{e}} \mathbf{F}_{1 \mathrm{e}}\right)$,

then these are sufficient conditions for (A3) to hold Klisch (2006b).

\section{Appendix B: Polyconvex collagen and proteoglycan strain energy functions}

In this appendix, we briefly outline the constitutive theory used in this paper to derive stress-strain functions for the collagen and proteoglycan constituents. Due to the observed tension-compression asymmetry in cartilage, we use a bimodular stress constitutive equation for the collagen constituent. For infinitesimal strains, Ateshian and colleagues (Soltz and Ateshian 2000; Wang et al. 2003) have employed bimodular stress constitutive equations that allow for different mechanical properties in tension and compression. Those models were based on a bimodular theory for infinitesimal strains (Curnier et al. 1995) in which the material constants may be discontinuous (or jump) across a surface of discontinuity in strain space, provided that stress continuity conditions are satisfied at the surface. Recently, the theory of Curnier et al. (1995) was generalized to second-order elasticity by Klisch (2006a). Here, we briefly describe the extension of these theories to the present application. In order to satisfy material stability restrictions, the strain energy functions used are polyconvex; see, for example, Itskov (2004) and Schroder et al. (2005).

The Cauchy and second Piola-Kirchhoff stress tensors (denoted as $\mathbf{T}$ and $\mathbf{S}$, respectively) are related by

$J \mathbf{T}=\mathbf{F S F}^{\mathrm{T}}$,

where $J=\operatorname{det} \mathbf{F}$. The stress constitutive equations for a Greenelastic material may be expressed as

$\mathbf{S}=2 \frac{\partial W}{\partial \mathbf{C}}$,

where $\mathbf{C}=\mathbf{F}^{\mathrm{T}} \mathbf{F}$ is the right Cauchy-Green deformation tensor and $\mathrm{W}$ is a scalar strain energy function that depends on a set of invariants of $\mathbf{C}$ corresponding to the material symmetry group. The fourth-order elasticity tensor is defined as

$C=\frac{\partial \mathbf{S}}{\partial \mathbf{C}}$. 
A scalar valued function of $\mathbf{C}$ that identifies a surface of discontinuity in the six-dimensional strain space of $\mathbf{C}$ is defined as

$g(\mathbf{C})=\mathbf{0}$,

and is restricted to be a function of the invariants corresponding to the material symmetry group. The stress function $\mathbf{S}$ may be different on either side of the surface of discontinuity; here we define

$\mathbf{S}=\mathbf{S}_{+} \quad$ if $g(\mathbf{C})>0, \quad \mathbf{S}=\mathbf{S}_{-} \quad$ if $g(\mathbf{C})<0$.

In a similar fashion, we can define $\left(W_{+}, W_{-}, C_{+}, C_{-}\right)$on different sides of the surface of discontinuity. In order to ensure that the stress-strain equation will be continuous across the surface of discontinuity, we must satisfy the following (necessary and sufficient) conditions (Curnier et al. 1995; Klisch 2006a):

$\mathbf{S}=\mathbf{S}_{+}=\mathbf{S}_{-}, \quad[[C]]=C_{+}-C_{-}=s(\mathbf{C}) \mathbf{M}(\mathbf{C}) \otimes \frac{\partial g}{\partial \mathbf{C}}$,

for all $\mathbf{C}$ that satisfy $g(\mathbf{C})=0$, where $[[C]]$ represents the jump in the elasticity tensor, $s(\mathbf{C})$ is a scalar valued function of $\mathbf{C}$, and $\mathbf{M}(\mathbf{C})$ is an arbitrary second-order tensor function of $\mathbf{C}$.

In this paper, we assume that the collagen constituent is a bimodular isotropic material with a compressible NeoHookean strain energy function:

$W^{\mathrm{c}}=\frac{1}{2} \mu I_{1}+\frac{1}{4} \lambda I_{3}-\left(\mu+\frac{1}{2} \lambda\right) \ln \sqrt{I_{3}}$

where $I_{1}=\operatorname{tr} \mathbf{C}, I_{3}=\operatorname{det} \mathbf{C}$, and $\mu$ and $\lambda$ are material constants. The bracketed term in Eq. (B7) is chosen to ensure a stress-free reference configuration. Also, we use the following surface of discontinuity:

$g(\mathbf{C})=I_{3}-1=0$,

so that the collagen constituent is bimodular with respect to volume change from the reference configuration. Consideration of the continuity conditions (B6) and straightforward calculations reveals that the only material constant that may jump across the surface (B8) is $\lambda$; we adopt the notation

$\lambda\left[I_{3}\right]=\lambda_{+} \quad$ if $I_{3}>1, \quad \lambda\left[I_{3}\right]=\lambda_{-} \quad$ if $I_{3}<1$.

Note that this correlates with the definition given in the body of the paper since $I_{3}=(J)^{2}$. Consequently, the bimodular Cauchy stress constitutive equation for the collagen constituent becomes

$\mathbf{T}^{\mathrm{c}}=\frac{1}{J}\left\{\mu(\mathbf{B}-\mathbf{I})+\frac{1}{2} \lambda\left[I_{3}\right]\left(J^{2}-1\right) \mathbf{I}\right\}$

where $\mathbf{C}=\mathbf{F}^{\mathrm{T}} \mathbf{F}$ is the right Cauchy-Green deformation tensor.
For the proteoglycan constituent, we assume the strain energy function

$W^{\mathrm{p}}=-\frac{1}{2} \alpha_{1} \ln I_{3}+\alpha_{2}\left(I_{3}\right)^{-1 / 2}$,

where $\alpha_{1}$ and $\alpha_{2}$ are material constants. Consequently, the Cauchy stress constitutive equation for the proteoglycan constituent becomes

$\mathbf{T}^{\mathrm{p}}=\left\{-\frac{\alpha_{1}}{J}-\frac{\alpha_{2}}{J^{2}}\right\} \mathbf{I}$.

The material constants were chosen based on an earlier study (Klisch et al. 2003) and are listed in Table 1. In the solid matrix stress-free reference configuration (i.e., $\mathbf{T}^{\mathrm{s}}=\mathbf{T}^{\mathrm{c}}+$ $\mathbf{T}^{\mathrm{p}}=\mathbf{0}$ ), these material constants correspond to constituent pre-stresses of $\mathbf{T}^{\mathrm{c}}=0.055 \mathrm{I} \mathrm{MPa}$ and $\mathbf{T}^{\mathrm{p}}=-0.055 \mathrm{I} \mathrm{MPa}$, a spherical collagen pre-strain (i.e., $\mathbf{F}_{\mathrm{o}}^{\mathrm{c}}=1.05 \mathrm{I}$ ), and coincides with the reference configuration for the proteoglycan constituent (i.e., $\mathbf{F}_{\mathrm{o}}^{\mathrm{p}}=\mathbf{I}$ ). Furthermore, the proteoglycan stress becomes unbounded as $J$ approaches 0 and approaches $\mathbf{0}$ as $J$ approaches infinity, based on the assumption that the proteoglycan stress is always compressive.

\section{References}

Argoubi M, Shirazi-Adl A (1996) Poroelastic creep response analysis of a lumbar motion segment in compression. J Biomech 29(10): 1331-9

Ateshian GA, Warden WH, Kim JJ, Grelsamer RP, Mow VC (1997) Finite deformation biphasic material properties of bovine articular cartilage from confined compression experiments. J Biomech 30:1157-64

Atkin RJ, Craine RE (1976) Continuum Theories of Mixtures: Basic Theory and Historical Development. Q J Mech Appl Math 29:209_ 244

Beaupre GS, Orr TE, Carter DR (1990) An approach for time-dependent bone modeling and remodeling - theoretical development. J Orthop Res 8:651-661

Breward CJ, Byrne HM, Lewis CE (2003) A multiphase model describing vascular tumour growth. Bull Math Biol 65(4):609-40

Buckwalter JA, Mankin HJ (1997) Articular cartilage. Part I: tissue design and chondrocyte-matrix interactions. J Bone Joint Surg Am 79-A:600-11

Buckwalter JA, Mankin HJ (1998) Articular cartilage repair and transplantation. Arthritis Rheum 41:1331-42

Buschmann MD, Kim YJ, Wong M, Frank E, Hunziker EB, Grodzinsky AJ (1999) Stimulation of aggrecan synthesis in cartilage explants by cyclic loading is localized to regions of high interstitial fluid flow. Arch Biochem Biophys 366:1-7

Carter DR, Wong M (1988) Mechanical stresses and endochondral ossification in the chondroepiphysis. J Orthop Res 6(1):148-54

Chahine NO, Wang CC, Hung CT, Ateshian GA (2004) Anisotropic strain-dependent material properties of bovine articular cartilage in the transitional range from tension to compression. J Biomech 37:1251-1261

Chen YC, Hoger A (2000) Constitutive functions for elastic materials in finite growth and deformation. J Elast 59:175-193

Chuong CJ, Fung YC (1986) On Residual Stresses in Arteries. ASME J Biomech Eng 120:382-388 
Cowin SC (1993) Bone stress adaptation models. J Biomech Eng 115:528-533

Cowin SC, Hegedus DM (1976) Bone remodeling I: A theory of adaptive elasticity. J Elasticity 6:313-325

Curnier A, He QC, Zysset P (1995) Conewise linear elastic materials. J Elasticity $37: 1-38$

Davisson TH, Sah RL, Ratcliffe AR (2002) Perfusion increases cell content and matrix synthesis in chondrocyte three-dimensional cultures. Tissue Eng 8:807-816

DiCarlo A, Quiligotti S (2002) Growth and balance. Mech Res Commun 29:449-456

Epstein M, Maugin GA (2000) Thermomechanics of volumetric growth in uniform bodies. Int J Plast 16:951-978

Ficklin T, Thomas G, Chen A, Sah R, Davol A, Klisch S (2006) Development of an experimental protocol to measure anisotropic material properties of bovine articular cartilage. 2006 Summer Bioengineering Conference, Amelia Island, Florida, ASME

Ganghoffer J-F, Haussy B (2005) Mechanical modeling of growth considering domain variation. Part 1: constitutive framework. Int J Solids Struct 42(15):4311-4337

Garikipati K, Arruda EM, Grosh K, Narayanan H, Calve S (2004) A continuum treatment of growth in biological tissue: the coupling of mass transport and growth. J Mech Phys Sol 52(7): $1595-1625$

Guilak F, Sah RL, Setton LA (1997) Physical regulation of cartilage metabolism. Basic orthopaedic biomechanics. Mow VC, Hayes WC (eds) Raven Press, New York 179-207

Hall AC, Urban JPG, Ellory JC (1988) Differential effects of steady and cyclic high hydrostatic pressures on protein and proteoglycan synthesis in cartilage. Trans Orthop Res Soc 13:71

Huang Z (2004) The equilibrium equations and constitutive equations of the growing deformable body in the framework of continuum theory. Int J Non-Linear Mech 39(6):951-962

Humphrey JD, Rajagopal KR (2002) A constrained mixture model for growth and remodeling in soft tissues. Math Model Meth Appl Sci 12(3):407-430

Hunziker EB (2001) Articular cartilage repair: basic science and clinical progress. A review of the current status and prospects. Osteoarthritis Cartilage 10:432-463

Itskov MAN (2004) A class of orthotropic and transversely isotropic hyperelastic constitutive models based on a polyconvex strain energy function. Int J Solids Struct 41:3833-3848

Kim YJ, Bonassar LJ, Grodzinsky AJ (1995) The role of cartilage streaming potential, fluid flow and pressure in the stimulation of chondrocyte biosynthesis during dynamic compression. J Biomech 28:1055-1066

Klisch SM (2006a) A bimodular theory for finite deformations: comparison of orthotropic second-order and exponential stress constitutive equations for articular cartilage. Biomech Model Mechanobiol 5:90-101

Klisch SM (2006b) Continuum models of growth with special emphasis on articular cartilage. Mechanics of Biological Tissue. Holzapfel GA, Ogden RW Springer, Berlin 119-133

Klisch SM, Hoger A (2003) Volumetric growth of thermoelastic materials and mixtures. Math Mech Solids 8:377-402

Klisch SM, Lotz JC (2000) A special theory of biphasic mixtures and experimental results for human annulus fibrosus tested in confined compression. J Biomech Eng 122:180-188

Klisch SM, Sah RL, Hoger A (2000) A growth mixture theory for cartilage. Mechanics in biology. Casey J, Bao G, ASME. AMB 242 \& BED 46:229-242

Klisch SM, Van Dyke T, Hoger A (2001) A theory of volumetric growth for compressible elastic materials. Math Mech Solids 6:551-75

Klisch SM, Chen SS, Sah RL, Hoger A (2003) A growth mixture theory for cartilage with applications to growth-related experiments on cartilage explants. J Biomech Eng 125:169-179
Klisch SM, Asanbaeva A, Sah RL, Davol A (2005a) Cartilage growth mixture model: finite strain theory, constitutive equations, and boundary-value problem solutions. International symposium on plasticity

Klisch SM, Sah RL, Hoger A (2005b) A cartilage growth mixture model for infinitesimal strains: solutions of boundary-value problems related to in vitro growth experiments. Biomech Model Mechanobiol 3(4):209-23

Klisch SM, Asanbaeva A, Sah RL, Davol A (2006) Theoretical and experimental study of articular cartilage growth. US national congress of theoretical and applied mechanics, Boulder

Kuhl E, Steinmann P (2003) Mass- and volume-specific views on thermodynamics for open systems. Proc R Soc London 459A:25472568

Lai WM, Mow VC, Roth V (1981) Effects of nonlinear strain-dependent permeability and rate of compression on the stress behavior of articular cartilage. J Biomech Eng 103:61-66

Lappa M (2005) A CFD level-set method for soft tissue growth: theory and fundamental equations. J Biomech 38(1):185-90

Lubarda VA, Hoger A (2002) On the mechanics of solids with a growing mass. Int J Solids Struct 39:4627-4664

Maroudas A, Venn M (1977) Chemical composition and swelling of normal and osteoarthrotic femoral head cartilage. II. Swelling. Ann Rheum Dis 36(5):399-406

Menzel A (2005) Modelling of anisotropic growth in biological tissues: a new approach and computational aspects. Biomech Model Mechanobiol 3(3):147-171

Mow VC, Ratcliffe A (1997) Structure and function of articular cartilage and meniscus. Basic orthopaedic biomechanics. Mow VC, Hayes WC Raven Press, New York pp 113-178

Mow VC, Kuei SC, Lai WM, Armstrong CG (1980) Biphasic creep and stress relaxation of articular cartilage in compression: theory and experiment. J Biomech Eng 102:73-84

Nieminen MT, Toyras J, Rieppo J, Hakumaki JM, Silvennoinen J, Helminen HJ, Jurvelin JS (2004) Quantitative MR microscopy of enzymatically degraded articular cartilage. Magn Reson Med 43:676-81

Nissi MJ, Toyras J, Laasanen MS, Rieppo J, Saarakkala S, Lappalainen R, Jurvelin JS, Nieminen HJ (2004) Proteoglycan and collagen sensitive MRI evaluation of normal and degenerated articular cartilage. J Orthop Res in press

Pazzano D, Mercier KA, Moran JM, Fong SS, DiBiasio DD, Rulfs JX, Kohles SS, Bonassar LJ (2000) Comparison of chondrogensis in static and perfused bioreactor culture. Biotechnol Prog 16(5):8936

Preziosi L, Farina A (2002) On darcy's law for growing porous media. Int J Non-Linear Mech 37:485-491

Quiligotti S (2002) On bulk growth mechanics of solid-fluid mixtures: kinematics and invariance requirements. Theor Appl Mech 2829:277-288

Rodriguez EK, Hoger A, McCulloch AD (1994) Stress-dependent finite growth in soft elastic tissues. J Biomech 27(4):455-467

Sah RL, Kim YJ, Doong JH, Grodzinsky AJ, Plaas AHK, Sandy JD (1989) Biosynthetic response of cartilage explants to dynamic compression. J Orthop Res 7:619-636

Schroder J, Neff P (2003) Invariant formulation of hyperelastic transverse isotropy based on polyconvex free energy functions. Int $\mathrm{J}$ Solids Struct 40:401-445

Schroder J, Neff P, Balzani D (2005) A variational approach for materially stable anisotropic hyperelasticity. Int J Solids Struct 42:4352-4371

Simon BR (1992) Multiphase poroelastic finite element models for soft tissue structures. Appl Mech Rev 45:191-218

Skalak R (1981) Growth as a finite displacement field. Proceedings of the IUTAM symposium on finite elasticity, Martinus Nijhoff, The Hague 
Skalak R, Zargaryan S, Jain RK, Netti PA, Hoger A (1996) Compatability and the genesis of residual stress by volumetric growth. J Math Biol 34:889-914

Skalak R, Farrow DA, Hoger A (1997) Kinematics of surface growth. J Math Biol 35(8):869-907

Smith GD, Knutsen G, Richardson JB (2005) A clincial review of cartilage repair techniques. J Bone Joint Surg 87B(4):445-449

Soltz MA, Ateshian GA (2000) A conewise linear elasticity mixture model for the analysis of tension-compression nonlinearity in articular cartilage. J Biomech Eng 122:576-86

Taber L (1998) A model for aortic growth based on fluid shear and fiber stresses. J Biomech Eng 120:348-354
Taber LA, Eggers DW (1996) Theoretical study of stress-modulated growth in the aorta. J Theor Biol 180:343-57

Volokh KY (2004) A simple phenomenological theory of tissue growth. Mech Chem Biosyst 1(2):147-160

Wang CC, Chahine NO, Hung CT, Ateshian GA (2003) Optical determination of anisotropic material properties of bovine articular cartilage in compression. J Biomech 36(3):339-53

Wu JZ, Herzog W, Epstein M (1998) Evaluation of the finite element software ABAQUS for biomechanical modelling of biphasic tissues. J Biomech 31:165-9 